\title{
Chapter 5-Summary
}

This chapter has concentrated on why, under certain circumstances and in certain disease states, we experience altered sensations in our gut, most commonly in the form of pain. In the first paper, Gerald Gebhart has posed several questions. Are there visceral pain receptors (nociceptors) in the gut and do the visceral afferent fibres linking these receptors to the spinal cord become sensitised in some way so that hyperalgesia is the result? Balloon distension experiments, which are considered to produce a reliable stimulus to hollow viscera, have been used in both humans and animals to explore these questions. Sensory innervation of the gastrointestinal tract involves all layers - that is, mucosa, muscle, and serosa. It would appear that visceral receptors are polymodal in character (that is, they respond to different kinds of stimuli). They appear to be chemosensitive, thermosensitive, and mechanosensitive. The visceral afferent neurones leading from these receptors pass through or near to prevertebral and paravertebral ganglia on their way to the spinal cord and at this point they can give off collateral axons which influence autonomic ganglion cell bodies, and consequently secretory and motor functions. There are two types of afferent fibre - one that responds to low pressure stimulation and one that responds only to high pressure stimulation. The low threshold fibres are the nociceptors. In functional bowel disorders, both types of fibres become sensitised and interact to pass "messages" to the central nervous system, but what is the relationship between sensitisation and the development of hyperalgesia? It must be a peripheral input which changes the behaviour of the central neurones. Experimental evidence suggests that a period of two hours of intensive input can lead to the development of hyperalgesia. It seems that in conditions such as inflammation, mechanosensitive visceral sensory fibres become sensitised-that is, are more responsive to stimuli but that there is also a "switch on" of silent afferent fibres or "silent nociceptors", which only become mechanosensitive after visceral inflammation. These silent or sleeping afferents, which are normally unresponsive to acute noxious stimuli, have been found in all of the tissues studied and contribute to hyperalgesia.

In the second paper of this chapter, Fernando Cervero has examined visceral pain from a central perspective. Visceral pain has five unique characteristics which differentiate it from other pain: (i) it is not evoked from all viscera, (ii) it is not linked to visceral injury, (iii) it is referred to other locations, (iv) it is diffuse and poorly localised; and (v) it is accompanied by motor and autonomic reflexes. The first two properties are conferred by the functional properties of the peripheral receptors that innervate the different visceral organs. Many viscera are innervated by receptors whose activation does not evoke conscious perception - that is, they are not strictly speaking "sensory receptors". The remaining three properties of visceral pain relate to central organisation of visceral nociceptive mechanisms - there is no separate visceral sensory pathway and there is a low proportion of afferents compared with those of somatic origin. However, nociceptive afferent discharges in visceral afferents provoke profound central effects, particularly when prolonged noxious stimulation of the viscera occurs. This produces an increase in the excitability of viscerosomatic neurones in the spinal cord. However, this does not appear to be the frequency dependent increase in neuronal excitability (known as "wind up") which is commonly seen in somatic nociceptive systems. The increase in excitability may be due to properties of the neuronal network activated by the stimuli, for example release of certain transmitters or development of positive feedback loops between spinal and supraspinal structures. It is clear that central circuits are important, as pain is often referred to areas which are not damaged or inflamed, for example skin shows an enhanced response to pinching when the colon is inflamed. Thus the central mechanisms are dependent on peripheral afferent drives.

It is thought that the glutamate receptor subtype $N$-methyl-D-aspartate (NMDA) has a particular role in mediating persistent pain and hyperalgesia in the spinal cord. It seems that acute noxious stimulation of normal visceral tissue provokes intensive responses which involve NMDA receptors, whereas the mechanisms do not operate in somatic pathways unless there is enhanced peripheral input resulting from injury or inflammation. Recent experiments in mice indicate that both substance $\mathrm{P}$ and neurokinin 1 receptors play a major role in the perception of visceral pain, but not acute pain. This has been demonstrated by the ability of animals to develop long term reactions to painful stimuli. It is likely that the central nervous system plays the larger role in modifying responses to stimuli.

In conclusion, this chapter has served to highlight the complexity of not only the peripheral nervous circuitry of the enteric nervous system but also the central "modifying" circuits in the spinal cord which have a major influence on the location and degree of perception of visceral pain. 